\title{
VERSITA
}

Lykhota K.N., Kochin A.V., Petrychenko A.V., Mocanu S.I.

\section{Measurement of biofunctional muscle activity of the maxillofacial area in the treatment of mesial occlusion with myofunctional apoaratus}

Association of Ukrainian Orthodontics

\section{ABSTRACT}

The paper presents measurements of electromyographic (EMG) parameters in the study of 63 patients, aged 9-12 years during their treatment of dentoalveolar form mesial occlusion with myofunctional equipment. After treatment, the EMG figures closer to the norm.

Keywords: EMG research, mesial occlusion, myofunctional devices

Kostiantyn Lykhota

k.lykhota@lykhota.kiev.ua $+380(50) 4626464$

\section{Introduction}

The introduction of the new dental practice all the standard devices myofunctional action allows more reliably eliminate functional violations in sagittal anomalies of the maxillofacial area. Due to the special design features and material used to produce cell phones, it is possible more comfortable fix to this or any other orthodontic abnormalities [1,2].

The purpose of our research was to study the bio-potentials of muscles of the maxillofacial area as a method of objective evaluation of treatment mesial occlusion (dentoalveolar forms I, II severity) with myofunctional devices. 
Material and method

For the treatment of mesial occlusion, we used standard devices of myofunctional action such as: T4K, T4A, T4B, "Myobrace" and individually manufacturing elastoaligner.

To evaluate the effectiveness of devises we used different methods, including electromyographic research (EMG). Electromyographic studies (EMG) of the muscles of the maxillofacial area we studied by a miniature computer Electroneuromyography "Neuron-EMG-Micro" and the PC H.Logyx. We conducted 111 surveys before and after the treatment myofunctional devices [1]. The control group consisted of 12 volunteers.

The quantitative data processing performed by using the computer program of [3].

\section{Results and discussions}

Table I - The mean values of the parameters of electromyographic muscle maxillofacial region in patients with mesial occlusion

\begin{tabular}{|c|c|c|c|c|}
\hline Parameters & $\begin{array}{l}\text { Norm } \\
(\mathbf{M}+/-)\end{array}$ & $\begin{array}{c}\text { Mesial occlusion } \\
(\mathbf{M}+/-\mathbf{m})\end{array}$ & $\mathbf{D}+/-\mathbf{m d}$ & p \\
\hline \multicolumn{5}{|c|}{ Temporalis muscle } \\
\hline $\mathrm{A}$ & $491.1+10.43$ & $259.29+25.41$ & $209.17+27.4$ & $<0.001$ \\
\hline $\mathrm{T}_{\mathrm{A}}$ & $0.35+0,01$ & $0.25+0.02$ & $0.10+0.02$ & $>0.005$ \\
\hline $\mathrm{T}_{\mathrm{C}}$ & $0.30+0.01$ & $0.26+0.03$ & $0.06+9.04$ & $>0.005$ \\
\hline $\mathrm{B}_{\mathrm{P}}$ & $266.9+11.71$ & $116.13+15.51$ & $150+77$ & $<0.001$ \\
\hline \multicolumn{5}{|l|}{ Masseter } \\
\hline $\mathrm{A}$ & $402.9+10.0$ & $223.91+27.03$ & $178.99+27.82$ & $<0.001$ \\
\hline $\mathrm{T}_{\mathrm{A}}$ & $0.36+0.01$ & $0.28+0.03$ & $0.08+0.03$ & $>0.005$ \\
\hline $\mathrm{T}_{\mathrm{C}}$ & $0.29+0.01$ & $0.36+0.03$ & $0.07+0.03$ & $>0.005$ \\
\hline $\mathrm{B}_{\mathrm{P}}$ & $227.3+10.57$ & $107.96+18.26$ & $119.89+21.10$ & $<0.001$ \\
\hline \multicolumn{5}{|c|}{ Mentohyoid muscle } \\
\hline $\mathrm{A}$ & $91.2+4.86$ & $160.1+22.6$ & $68.9+23.6$ & $>0.005$ \\
\hline $\mathrm{T}_{\mathrm{A}}$ & $0.28+0.01$ & $0.29+0.02$ & $0.01+0.02$ & $>0.005$ \\
\hline $\mathrm{T}_{\mathrm{C}}$ & $0.26+0.01$ & $0.32+0.3$ & $0.06+0.03$ & $>0.005$ \\
\hline $\mathrm{B}_{\mathrm{p}}$ & $55.14+3.71$ & $69.34+11.2$ & $14.2+11.3$ & $>0.005$ \\
\hline
\end{tabular}


$150+77$ units $(\mathrm{P}<0,001)$. The coefficient for the coordination of one chewing motion is reduced by $25 \%(\mathrm{P}<0.001)$, and the coefficient of coordination for the whole chewing period increased by $36.8 \%$ (P $<0.001)$. As for mentohyoid muscle, then there is a steady tendency to increase their biological activity by $68.9+23.6 \mathrm{mkV}(\mathrm{P}<0.05)$. The increase in biopotentials and performance of their duration are not significant, although the action potential increased by 14.2 units and amounts to $25.8 \%$ of normal.

To determine the possible impact of the muscles of the maxillofacial area the size of the jawbones, we conducted a correlation analysis of the indicators of chewing EMG, temporal and mentohyoid muscles, both among themselves and with the parameters of the facial part of the skull from data on the side of the head TWG patients with sagittal anomalies. These relationships can affect not only on the results of orthodontic treatment of sagittal anomalies, but also on the likelihood of its recurrence. The high relapse rate might be related to dysfunction of the masticatory muscles.

Table II - The correlation coefficient between the parameters of EMG maxillo-facial area.

\begin{tabular}{|l|c|c|c|}
\hline \multirow{2}{*}{ Parameters } & \multicolumn{3}{|c|}{ Muscles } \\
\cline { 2 - 4 } & $\begin{array}{c}\text { Temporal / } \\
\text { Chewing }\end{array}$ & $\begin{array}{c}\text { Temporal / } \\
\text { Mentohyoid } \\
\text { muscle }\end{array}$ & $\begin{array}{c}\text { Chewing / } \\
\text { Mentohyoid } \\
\text { muscle }\end{array}$ \\
\hline $\begin{array}{l}\text { EMG } \\
\text { amplitude }\end{array}$ & 0.46 & 0.43 & 0.88 \\
\hline $\mathrm{T}_{\mathrm{A}}$ & 0.84 & 0.54 & 0.64 \\
\hline $\mathrm{T}_{\mathrm{C}}$ & 0.82 & 0.77 & 0.56 \\
\hline $\mathrm{B}_{\mathrm{P}}$ & 0.69 & 0.1 & 0.87 \\
\hline
\end{tabular}

As can be seen from table II, all the correlation was positive. For the masseter and temporalis muscles of the positive relationship between the amplitudes and biopotentials were average, while the relationship between the parameters of duration was strong. Between the same parameters and the temporal muscle mentohyoid communications were of medium strength. Between themselves biopotentials, correlation was very weak. In contrast, the relationship between the amplitudes of EMG and biopotentials chewing and mentohyoid muscle is high, and the between indicators of duration is average.

The length of the body of the upper jaw acts EMG amplitude and action potential proper chewing, temporal and mentohyoid muscles and reverse the high and the average correlation. Thus, the increased electrical activity of these muscles affects the length of the body and can contribute to reduction of activity elongated body of the mandible.

Consequently, it can be assumed that using myofunctional apparatus, growth of the upper jaw, not only facilitates removal of soft tissue pressure cheek and bioelectric activity and decreased masticatory muscles.

The size of the lower jaw - the length of the body (Po Go) and the height of their appendages (Co Go) also depend on the functional activity of the muscles.

Thus, analyzing the data, we can conclude that in patients with mesial occlusion significantly reduced the activity of masseter and temporalis muscles, raising the lower jaw and the elevated electrical activity mentohyoid muscles that lower the mandible. The coefficients coordination antagonist muscles and synergists changed. The coefficient for the coordination of one chewing motion is reduced by $25 \%$ ( $\mathrm{P}<0.001)$, and the coefficient of coordination for the entire chewing period increased by $36.8 \%$ ( $\mathrm{P}$ $<0.001$ ).

Our data matches with data of [4]. To evaluate the treatment of mesial occlusion (I-st, II-nd severity forms dentoalveolar) for the same parameters studied biofunctional activity of the muscles of the maxillofacial region. The results showed a statistically significant improvement in the results of the functional state of the muscles. The functional activity of the dentition muscles is approaching the normal.

Thus, the electromyographic research (EMG) showed that the use of myofunctional devices (T4K, T4A, T4B, "miobrace", I-3, and others) allows to avoid more pressure masticatory muscles lips, change the position of the tongue, thereby contributing to the growth and the development of the upper jaw, the correct position of the mandible. 
3. Dvornik V.N. (1999). Computer electromyographic evaluation standards masticatory muscles. Stomat. 391-392

1. Lykhota K. N. (2008). Short and long term results of treatment of dentofacial anomalies using myofunctional devices. Mat. III (A) Congress Assn. Stom. Poltava, Ukraine. 479-480

4. Gioeva Y.A., Polma L.V. \& Gordina E.S. (2003). Changes to the profile of the person as a result of orthodontic treatment mesial occlusion. Mater. VII IU. Conf. maxillofacial surgeons and dentists. 49-51

2. Persin L.S. (1999). Orthodontics. Diagnosis. Types of dentition anomalies. Second ed. Moscow: Ortodent-Info 